http://jmscr.igmpublication.org/home/ ISSN (e)-2347-176x ISSN (p) 2455-0450

crossref DOI: https://dx.doi.org/10.18535/jmscr/v8i2.68

Journal Of Medical Science And Clinical Research

\title{
Comparative Study of Accelerated Ponseti Cast with Standard Ponseti Cast
}

\author{
Authors \\ Dr Malik Naseer Ahmad, Dr Abdul Ghani, Dr Rajinder Singh, \\ Dr Mohammad Farooq Bhatt, Dr Sheikh Aisha Shabir, Dr Khalid Nisar, \\ Dr Tanveer Ah Bhatt, Dr Imtiyaz Ah Beigh
}

\begin{abstract}
Introduction: Congenital talipes equinovarus (CTEV) commonly known as Clubfoot is a common birth defect that affects one in thousand live births. In past attempt at correction, based on forceful manipulations, resulted in incomplete corrections as well as iatrogenic deformities. With the advancement in understanding of kinematics and pathoanatomy of the CTEV, the Ponseti developed a novel method of correction, which has gained wide acceptance worldwide. Various modification have been done in terms of frequency of casting with evidence to suggest that accelerated frequency of cast changes has comparable outcomes to those of the conventional Ponseti method. Our study was undertaken to compare the results of Standard Ponseti method with that of the Accelerated Ponseti method in management of idiopathic CTEV.

Method \& Material: Only idiopathic cases of both gender less than 1 year age were included in doubleblind randomized prospective comparative study was conducted in the Department of Orthopaedics of Govt Medical College and Hospital, 55 patients with total 80 clubfeet, who met the inclusion criteria were included in the study.

Result: In our study of 55 patients with 40 idiopathic clubfeet in each group. The two groups were almost comparable with Pirani score of 4.69 in accelerated group and 4.88 in standard group respectively at the initiation of the casting. Mean duration of treatment from the first cast to tenotomy in accelerated ponseti group was 2.21 weeks and in standard ponseti group was 5.8 weeks respectively. Post cast Pirani score was 0.48 in accelerated group and 0.5 in standard group respectively. All the patient were followed for an average period of 15 month (12-18 month). No major complications were recorded in any group. The two study groups, the traditional and the accelerated Ponseti groups had nearly equivalent results with significant reduction in the correction time in the accelerated Ponseti group.

Conclusion: The clubfoot in developing countries has social stigma, the early and the promising result of the accelerated method of ponseti casting has a dramatic impact on both parents and the treating orthopaedician. The accelerated ponseti casting has remarkably reduced the overall duration of the treatment of Ponseti casting without any complication. It has reduced the overall economic burden on the poor patient by reducing the number of days of work lost as well as the total expenditure on the travel.
\end{abstract}

\section{Introduction}

Clubfoot also known as congenital talipes equinovarus (CTEV), a common birth defect that affects one in thousand live births ${ }^{[1]}$. To achieve a functional, plantigrade foot, enabling the patient to wear usual shoes, and to prevent arthritic degenerations in adulthood without surgical correction has been the primary goal of treatment $^{[2]}$. Sir Hugh Owen Thomas developed Thomas wrench to attempt correction which was 
based on forceful manipulations, however this resulted in incomplete corrections as well as iatrogenic deformities ${ }^{[3]}$. Kite in 1939, attempted to correct the club foot with method of gentle manipulation and serial casting ${ }^{[4,5]}$. however there was a fundamental flaw in technique, this method of correction was often quite lengthy with children being casted for up to 2 years. In addition, because of incomplete corrections , $50 \%$ to $75 \%$ the of patients still required soft tissue release surgery ${ }^{[6]}$. With the advancement in understanding of kinematics and pathoanatomy of the club foot. The Ponseti developed a novel method of correction, which has gained wide acceptance worldwide by producing good long- term results ${ }^{[7,8]}$. The standard Ponseti method uses serial application of weekly above knee plaster casts to gradually correct the deformity. The researchers have modified the frequency of casting, with strong evidence to suggest that accelerated frequency of cast changes has comparable outcomes to those of the conventional Ponseti method ${ }^{[9-11]}$. Our study was undertaken to compare the results of Standard Ponseti method with that of the Accelerated Ponseti method in management of idiopathic CTEV.

\section{Methods and Material}

This double-blind randomized prospective comparative study was conducted in the Department of Orthopaedics of Govt Medical College and Hospital, 55 patients with total 80 clubfeet, who met the inclusion criteria were included in the study. Only idiopathic cases of both gender less than 1 year were included. Syndromic child, relapsed, neglected, resistant and recurrent cases were excluded. After a, thorough clinical examination and confirmation of diagnosis, Severity was assessed by modified Pirani scoring system. Each parameter was scored according to the Modified Pirani Score (table 1). Six clinical signs, each scored 0 (normal), 0.5 (mildly abnormal) or 1 (severely abnormal) respectively. Thus, each foot can receive a Midfoot score between 0-3 and a Hindfoot score between 0-3 and a total score between 0-6

Table 1

\begin{tabular}{|l|c|c|c|}
\hline HIND FOOT SCORE & MILD & MODERATE & SEVERE \\
\hline Posterior crease & 0 & 0.5 & 1 \\
\hline Rigid Equinus & 0 & 0.5 & 1 \\
\hline Empty heel & 0 & 0.5 & 1 \\
\hline MID FOOT SCORE & & & \\
\hline Curved lateral border & 0 & 0.5 & 1 \\
\hline Medial foot crease & 0 & 0.5 & 1 \\
\hline Talus head coverage & 0 & 0.5 & 1 \\
\hline
\end{tabular}

Manipulation of foot and long leg plaster of paris application was done with the child comfortably placed in mothers lap. On each cast removal improvement in pirani score was noted. In all patients, the cavus is corrected first by extending the first metatarsal and supinating the forefoot. This elevates the first ray and puts the forefoot in proper alignment with the hindfoot. In second the varus and adduction of midfoot was corrected by abducting the supinated foot with counter pressure applied with the thumb against the head of the talus. Manipulation and cast was stopped when midfoot and hindfoot scores were zero with 70 degrees of abduction of the forefoot. With abduction of 70 degrees if dorsiflexion was less than 10 degrees then percutaneous tenotomy of the Achilles tendon was performed. All cases were done in operation theatre under local anesthesia as an outpatient procedure. Patients were monitored for 1 hour post operatively. A long leg cast was applied in 70 degrees of abduction and maximum available dorsiflexion immediately after tenotomy and maintained for further 3 weeks to allow healing of the tendon. After 3 weeks cast was removed and steenbeek abduction brace was applied.

Number of cast and days of treatment noted were before the last cast for 3 weeks. Children were reviewed every month and Pirani scores were documented. Foot abduction brace was worn for 23 hours during first three months after casting and then at night until child is about 4 years old. Foot-abduction brace is used to maintain the correction. This brace consists of a bar with shoes attached at the ends at 70 degrees of outward rotation on the affected side and 40 degrees on the normal side. The length of the bar should be equal 
to the width of the child's shoulders. Parent selfreport on brace wear was used to assess compliance. 55 patients with total 80 clubfeet who met the inclusion criteria were included: Age less than one year, unilateral or bilateral idiopathic CTEV, patients of either sex, parents of the child giving consent to take part in the study. Age more than one year, earlier treated with plaster cast application or any other method, earlier operated for clubfoot, atypical, syndromic or secondary clubfoot were excluded from the study. A computer generated randomisation plan was generated and accordingly, 27 with 40 clubfeet patients were treated with once weekly casting and 28 with 40 clubfeet patients were treated with twice weekly casting on fixed days. The cases were treated on an outpatient basis. All cases were clinically assessed using Pirani scoring system at initial presentation and subsequent visits. Ponseti method of casting was followed in both Standard and Accelerated Ponseti groups.

\section{Result}

In our study of 55 patients with 80 idiopathic clubfeet, the male to female ratio is 2.1 in standard Ponseti group and 1.54 accelerated group respectively. The mean age of the patient with clubfoot was 13.5 weeks in accelerated group and 16.8 weeks in standard group. The two groups were almost comparable with respect to age, sex and side of involvement .Each group with 40 feet with 28 patients in accelerated Ponseti and 27 patient in standard Ponseti group respectively. Pirani score before initiation of casting was 4.69 in accelerated group and 4.88 in standard group respectively. Mean duration of treatment from the first cast to tenotomy in accelerated ponseti group was 2.21 weeks and in standard ponseti group was 5.8 weeks respectively. Total number of plaster cast applied were 6.53 in accelerated Ponseti and 5.14 in standard Ponseti group respectively. Post cast Pirani score was 0.48 in accelerated group and 0.5 in standard group respectively. Tenotomy was required in $37(92.5 \%)$ feet in accelerated group and $34(85 \%)$ feet in standard group respectively. All the patient were followed for an average period of 15 month (12-18 month). At every follow up each foot was assessed for relapse. Recurrences with different degrees were observed at later follow-up in the form of forefoot adduction, heel varus and equinus, in $6(15 \%)$ feet in the accelerated Ponseti group and 5 feet in standard Ponseti group respectively. These relapsed feet were evaluated and completely corrected by either accelerated Ponseti or standard method depending on the assigned group. Four patients of the accelerated group and 2 patient of the standard group required tenotomy again. No major complications were recorded in any group. The two study groups, the traditional and the accelerated Ponseti groups had nearly equivalent results with significant reduction in the correction time in the accelerated Ponseti group.

\section{Discussion}

For the normal development of the foot in the patients with CTEV an early correction of all aspects of the deformity is essential. For deformity correction, the Ponseti technique is considered as the gold standard treatment for clubfoot $^{[12]}$. The ponseti technique is a specific method of manipulation, with head of talus as fulcrum to stretch the contracted ligaments and hold the stretch with serial casting followed by Achilles tendon tenotomy and Bracing-Foot abduction brace $e^{[13-15]}$. Keeping in view the psychological and economic burden over the family of the patient with club foot travelling long distances to the location of treatment centre mostly the tertiary care hospital, it has become a necessity to shorter the time required for deformity for the convenience of both the patient and the parents. The largest comprehensive study was undertaken over an 11 year period by Morcuende et al. in $2005^{[9]}$ by performing serial manipulations by changing the cast every 5 days to obtain a successful correction with or without minimal side effects ${ }^{[16]}$. In the study by Morcuende et al, 230 patients with 319 clubfeet were were allocated into standard Ponseti and 
accelerated group based on their distance from treatment centre. The accelerated ponseti group was treated with every fifth day cast changes while the standard ponseti group was manipulated and casted weekly cast changes. Their study showed average time from first cast to Achilles tenotomy was 16 days for the 5-day group and 24 days for the 7-day group ( $\mathrm{P}=0.001)$ which was remarkable. $\mathrm{Xu} \mathrm{RJ}^{[17]}$ performed accelerated Ponseti technique with casting twice a week without any complication and found that significant results were obtained in shorter period in patients with the accelerated group compared with those managed with the standard Ponseti casting. In our study tenotomy was required in $37(92.5 \%)$ feet in accelerated group and $34(85 \%)$ feet in standard group respectively comparable to the study by Radler et al. who reported a tenotomy rate of $(96 \%)$ and Porecha et al. $(96 \%)^{[18,19]}$. This characteristic was identified by Scher et al. ${ }^{[20]}$ who related higher Pirani scores to the need for a tenotomy. In our study after attaining the correction, parents were educated about the importance of the strict compliance the brace protocol of 23 hors for initial three month and then during the nap time only. relapse with different degrees were observed at later follow-up in the form of forefoot adduction, heel varus and equinus, in $6(15 \%)$ feet in the accelerated Ponseti group and $5(12.5 \%)$ feet in standard Ponseti group respectively which was relatively low as compared to the other studies. Ponseti reported a recurrence rate of $46 \%$, which was treated by remanipulation and casting, Achilles tenotomy or even by tibialis anterior tendon transfer. Failure of correction using the Ponseti is attributed mainly to lack of brace compliance, low parental educational level, severity of deformity and technical error in casting $^{[21]}$. Cosma and Vasilescu ${ }^{[22]}$ had a relapse rate of $19 \%$; they noticed that most of the relapses were encountered in the 1-4 years of age interval and considered that wearing braces is the best prevention for relapses. In our study after attaining the correction, parents were educated about the importance of the strict compliance to the brace protocol of 23 hours for initial three month and then during the nap time only. Relapse with different degrees were observed at later follow-up in the form of forefoot adduction, heel varus and equinus, in $6(15 \%)$ feet in the accelerated Ponseti group and $5(12.5 \%)$ feet in standard Ponseti group respectively which was relatively low as compared to the other studies.

\section{Conclusion}

The clubfoot in developing countries has social stigma though the cause is not known, still the culprit and the soft target has been the mother as far the Indian society is concerned. Looking at the tears rolling down and the deep pain both in terms of the concern for their child as well as the torture they go through, the early and the promising result of the accelerated method of ponseti casting has a dramatic impact on both parents as well as the treating orthopaedician. The accelerated ponseti casting has remarkably reduced the overall duration of the treatment of Ponseti casting without any complication. It has reduced the overall economic burden on the poor patients by reducing the number of days of work lost as well as the total expenditure on the travel. The satisfactory outcome can be measured in terms of the smiling face of the anxious parents by getting the normal looking foot in few weeks without any complications.

\section{Reference}

1. Dobbs MB, Gurnett CA. Update on clubfoot: etiology and treatment. Clinical orthopaedics and related research. 2009; 467(5):1146. https://doi.org/10.1007/s11999-009-07349 PMID: 19224303.

2. Cosma DI, Vasilescu DE. Ponseti treatment for clubfoot in Romania: a 9year single-centre experience. Journal of Pediatric Orthopaedics B. 2014;23(6):5126. 
3. Dobbs MB, Morcuende JA, Gurnett CA, Ponseti IV. Treatment of idiopathic clubfoot: an historical review. Iowa Orthop J. 2000;20:59-64.

4. Kite JH. Some suggestions on the treatment of club foot by casts. J Bone Joint Surg Am. 1963;45:406-412.

5. Kite JH. Principles involved in the treatment of congenital club-foot. 1939. J Bone Joint Surg Am. 2003;85:1847.

6. Lovell WW, Farley D. Treatment of congenital clubfoot. Ona J. 1979;6:453456.

7. Morcuende JA, Dolan LA, Dietz FR, Ponseti IV. Radical reduction in the rate of extensive corrective surgery for clubfoot using the Ponseti method. Pediatrics 2004;113:376- 80 .

8. Ponseti IV. Treatment of congenital club foot. J Bone Joint Surg Am 1992;74:448- 54.

9. Morcuende J, Abbasi D, Dolan L, Ponseti I. Results of an accelerated Punster protocol for clubfoot. J Pediatr Orthop 2005;25: 623-6.

10. Harnett P, Freeman R, Harrison WJ, Brown LC, Beckles V. An accelerated Ponseti versus the standard Ponseti method: a prospective randomised controlled trial. J Bone joint Surg [Br] 2011; 93(3): 404-408.

11. Sutcliffe A, Vaea K, Poulivaati J, Evans AM. 'Fast casts': Evidence based and clinical considerations for rapid Ponseti method. Foot and ankle online $j$ 20013;6(9)2.

12. Hennessey TA (2012) Congenital clubfoot and the Ponseti method: a review of recent literature. Curr orthop pract 23(5):442-447

13. Cosma DI, Vasilescu DE (2014) Ponseti treatment for clubfoot in Romania: a 9year single-centre experience. J Pediatr Orthop B 23(6):512-516. doi:10.1097/BPB.0000000000000081
14. Panjavi B, Sharafatvaziri A, Zargarbashi RH, Mehrpour S (2011) Use of the Ponseti method in the iranian population. J Pediatr Orthop 32:11-14

15. Scher DM, Feldman DS, van Bosse HJ, Sala DA, Lehman WB. Predicting the need for tenotomy in the Ponseti method for correction of clubfeet. J Pediatr Orthop 2004;24:349- 52.

16. Radler C, Mindler GT, Riedl K, Lipkowski C, Kranzl A (2013) Midterm results of the Ponseti method in the treatment of congenital clubfoot. Int Orthop 37:18271831

17. Porecha MM, Parmar DS, Chavda HR (2011) Mid-term results of Ponseti method for the treatment of congenital idiopathic club- foot-(a study of 67 clubfeet with mean five year follow-up). J OrthopSurg Res 6:3. doi:10.1186/1749-799X-6-3

18. Xu RJ. A modified Ponseti method for the treatment of idiopathic clubfoot: a preliminary report. Journal of Pediatric Orthopaedics. 2011; 31(3)317-319.

19. Cooper DM, Dietz FR. Treatment of idiopathic clubfoot: a thirty-year follow-up note. J Bone Joint Surg Am. 1995;77(10):1477-1489.

20. Laaveg S, Ponseti IV. Long-term results of treatment of congenital club foot. The Journal of Bone \&Joint Surgery. 1980;62(1):23-31.

21. Sana Ullah, Muhammad Inam, Muhammad Arif. Club foot management by accelerated Ponseti technique. http://www.scopemed.org/?mno=170854 15 J Bone Joint Surg Br. 2011; 93(3):4048. 